targeting type-I interferons. Using our validated bioinformatics pipeline and methodology, we have now identified two other cohorts of patients with genetic variants that impair thymic tolerance and T1-IFN signalling, respectively. Biochemical assays confirmed the variants impair protein function. Furthermore, flow cytometry identified immunophenotypes in the patients' PBMCs that may explain disease pathogenesis. The mechanisms by which they drive SLE pathogenesis are being evaluated in patient-specific mice mouse models.

Conclusions By understanding the precise genetic mechanisms that contribute to SLE pathogenesis, our data is able to stratify patients and, through a personalised approach, identify tailored therapeutic options.

\section{RELEVANCE OF MOUSE LUPUS MODELS OF LUPUS NEPHRITIS TO PROGRESSION OF CKD}

${ }^{1} \mathrm{CC}$ Berthier*, ${ }^{1} \mathrm{M}$ Kretzler, ${ }^{2} \mathrm{~A}$ Davidson. ${ }^{1}$ University of Michigan, Internal Medicine, Ann Arbour, USA; ${ }^{2}$ Feinstein Institute for Medical Research, Centre for Autoimmunity and Musculoskeletal Diseases, Manhasset- NY, USA

\subsection{6/lupus-2017-000215.41}

Background and aims Risk for progression of CKD in humans is associated with an interstitial molecular signature containing 68 genes. Of these, a decrease in renal expression of EGF with a concomitant increase in urinary EGF improves the ability to predict CKD expression.

Methods To determine whether these 68 genes can be used in pre-clinical studies to model disease and therapeutic responses, we analysed microarray data of kidneys from three mouse lupus strains at various disease stages and after remission induction. Renal macrophage gene expression was assessed using RNASeq.

Results 61/64 genes have mouse gene IDs and are represented on the mouse microarray chip. Of these 49 were regulated in the same direction as in humans in at least one mouse strain with 28 common to all three strains. 9/61 genes, including EGF and TIMP1 only became abnormally regulated during established disease or during complete proteinuric relapse, confirming their association with CKD progression. Renal C1qa is a CKD marker produced mainly by renal macrophages but has a similarly high expression level in isolated pre-nephritis and nephritic renal macrophages. It can therefore be used as a biomarker of increased macrophage infiltration, a known poor prognostic feature in human lupus nephritis.

Conclusions Mice with lupus nephritis have a similar pattern of CKD-related gene expression to humans and these genes can be used to track therapeutic responses. Downregulation of EGF and upregulation of TIMP1 indicate progressive disease and C1qa can be used as a marker of macrophage infiltration. The fibrosis signature is best modelled in NZW/BXSB mice.

\section{GENE EXPRESSION PROFILE FROM 1,760 SLE PATIENTS REVEALS NOVEL COMPLEX INTERFERON RESPONSIVE GENE NETWORKS}

${ }^{1}$ RW Hoffman, ${ }^{2}$ ER Dow*, ${ }^{2} \mathrm{NB}$ Perumal, ${ }^{3} \mathrm{GV}$ Rocha, ${ }^{3} \mathrm{E}$ Nantz, ${ }^{3} \mathrm{~N}$ Shaikh, ${ }^{2} \mathrm{~B}$ Steere, ${ }^{3} \mathrm{~B}$ Kechavarzi, ${ }^{2} \mathrm{RJ}$ Benschop, ${ }^{2}{ }^{3} \mathrm{RE}$ Higgs. ${ }^{1}$ Eli Lilly and Company, Immunology, Indianapolis, USA; ${ }^{2}$ Eli Lilly and Company, TTX, Indianapolis, USA; ${ }^{3}$ Eli Lilly and Company, Statistics, Indianapolis, USA

10.1136/lupus-2017-000215.42
Background and aims RNA profiling was performed on 1760 SLE patients from two, large Phase III clinical trials, ILLUMINATE-1 and -2. SLE was compared to both healthy controls and other autoimmune diseases, including rheumatoid arthritis, psoriasis and psoriatic arthritis. The goals of this study were to characterise gene expression networks in SLE using these large cohorts, and to compare gene expression phenotypes in SLE to healthy controls and other autoimmune diseases.

Methods Blood was collected at baseline and RNA was interrogated on all samples using Affymetrix HTA 2.0 microarrays and on select SLE samples using NanoString nCounter ${ }^{\mathrm{TM}}$. Complete demographics, serum IgG anti-dsDNA antibodies, and complement were measured in SLE. Analyses of gene expression and gene pathways were performed.

Results Baseline elevation of interferon responsive genes (IRG) was detected in SLE and associated with younger age, elevated anti-dsDNA antibodies, elevated SLEDAI and decreased levels of C3. Significant differences in SLEDAI organ domain involvement between IRG-positive and IRG-negative groups were observed. Elevated expression of IRG, genes involved with B cell and plasma cell biology, and with cell cycling and signalling were detected in SLE. A bimodal expression pattern of IRG was unique to SLE. Substantial heterogeneity of expression of IRG and complex relationships in interferon (IFN) gene networks were observed.

Conclusions There was substantial heterogeneity of gene expression in IFN gene networks when examining individual IFN genes and complex relationships were observed among IFN gene networks. Low IFI27 was identified as a novel subtype of IFN signature in SLE.

\section{Poster Session}

\section{Adaptive immunity and lymphocytes}

\section{ENHANCED IMMUNE CELL ACTIVITY BY KOREAN RED GINSENG IN PORCINE}

A Adithan, A Paulrayer, HS Jeong, JH Kim. Chonbuk National University, veterinary physiology, Iksan, Republic of Korea

\subsection{6/lupus-2017-000215.43}

Background and aims In search of immunomodulatory agents, natural products play a vital role since they have relatively low toxicity in clinical applications. Korean red ginseng (KRG) has been used in Korea, Japan, and china as a traditional medicine. KRG has proven for its efficacy against various human diseases such as cancer, diabetes, and atherosclerosis.

Methods In this study, KRG was assessed for its ability to act as an adjuvant for the immune response of porcine splenocytes.

The porcines were administered with different concentrations $(200$ and $400 \mathrm{mg} / \mathrm{kg} /$ day) of KRG, orally for 28 days.

Results The splenocytes isolated from KRG treated group showed enhanced immune response in a concentration dependent manner when compared to untreated porcine splenocytes. Further, the intracellular levels of perforin, Granzyme $B$ and NKG2D were found to be significantly increased in transcriptional and translational level as revealed by RT-PCR and western blot analysis respectively. In addition, we compared the cytotoxic ability of splenocytes treated with KRG against K-562 cell for 28 days. The KRG activated porcine 